\title{
LATE EFFECTS OF PENETRATING WOUNDS OF THE CHEST.
}

\author{
By C. PRICE THOMAS, F.R.C.S.
}

(Assistant Surgeon, Westminster Hospital and Brompton Hospital for Diseases of the Chest.)

In assessing the late effects of penetrating wounds of the chest, and for the purpose of this paper, late effects mean those suffered ro-20 years after, it would be helpful to group the cases roughly under two separate headings.

Firstly those cases in which the symptoms referable to the chest at the time of the original wound were relatively slight, this excludes symptoms due to shock, exposure and loss of blood. Many of these cases have through and through wounds, especially with high velocity missiles such as rifle bullets, without hæmoptyses or an accompanying hæmothorax, in some cases the foreign body is retained and there may have been no hæmoptysis or other complication, or if they were present they were so slight as to make little or no impression on the patient's mind. The second group are those in which there were severe symptoms referable to the thoracic contents, such as severe hæmoptysis, hæmothorax or infection either intrapulmonary or intrapleural. The reason for stressing the differentiation is to point out that the first group seem to be as liable to serious late effects as the second. In consequence the consideration of the early treatment of these cases should take this factor into account.

The late complications can conveniently be divided into three heads: (a) Those referable to the chest wall (b). Those to the cardiovascular system and (c) Those to the lungs and pleura.

Late complications referable to the mediastinum have not come under review. The reason probably being that if the patient survives a wound of the mediastinum, without infection either as a result of a wound either with or without a retained foreign body, then it is most unlikely that the future holds any serious menace, and if infection does occur at the time of injury the chances of survival with a mediastinitis of the type associated with war wounds is so slight as to be negligible.

The effects referable to the chest wall, without an underlying lesion.

(I) Pain. This is a not uncommon complaint. It occurs often when there is no discoverable intrathoracic lesion, and its occurrence cannot be questioned, even though it is a common complaint with the confirmed malingerer. The pain may have a definite anatomical distribution, either along one or two intercostal nerves, at the site of a wound entrance or exit, or in the distribution of the 3 rd, $4^{\text {th }}$ or $5^{\text {th }}$ cervical nerves when the diaphragm has been involved. Often however there seems to be no anatomical explanation for the distribution and although in this group one may suspect gross exaggeration of mild symptoms or frank malingering, it behoves one to be extremely careful before arriving at this opinion. In one case with an anomalous distribution of pain in the upper chest, where the lesion was confined to the lower lobe, the cause of the pain was eventually proved to be a carcinoma of the upper lobe. Pain of an anomalous character should be investigated thoroughly by all means at our disposal, clinical and radiographic, before arriving at a diagnosis.

(2) Mobility of chest wall. Mobility of the chest wall due to destruction of the bony framework, will, when the mediastinum is normal, give rise to mediastinal flutter, with its attendant disability and dangers. In the type of case under discussion the patient has weathered the initial storm and has managed to carry 
on for some years tolerating his disability. When the case presents himself at this date his chief and practically only complaint is dyspnœea which prevents any degree of exertion. Fixation of the chest wall by an external pad in these cases will often change the physio-mechanics of the chest sufficiently to allow the patient to carry on a normal life. There can be no doubt that these cases during the early years are able to carry on by virtue of a large vital capacity. Graham estimated that a man with a vital capacity of 7,000 c.cs. could tolerate an open pneumothorax of I5 square inches. Increase in age with its accompanying increase in the rigidity of the chest wall due to calcification of costal cartilages and arthritis of the costovertebral joints, weakening of the musculature and emphysema, all contribute to a decrease in vital capacity which make the chest wall deficiency a serious factor.

Late effects of wounds of the heart and vessels.

The only lesion which occurs at this late date as a result of cardiac wounds is an aneurysm of the heart. Sauerbruch states that this is more likely to occur after a wound in which spontaneous healing has taken place and that it is much less likely to occur in cases where primary suture has been performed.

This condition generally is only discovered during routine post mortem examination, or as a result of rupture and consequently needs no consideration as to treatment.

The pericardium is also very free from clinical lesions. Intrapericardial adhesions may be found post mortem, and adherent pericardium also if infection has occurred at the time of the original wound, but constrictive adhesive pericarditis has not been met with.

Wounds of the vessels in the thorax are similar to those met with in other parts of the body, but are much less common than in the peripheral vessels. Arterio-venous communication is the only one that has been met with, between the left common carotid and the jugular vein just within the upper aperture of the thorax. This condition, even though very rare, is doubtless more common than its clinical occurrence suggests for an arterio-venous communication as close to the heart as the main aortic branches will fairly rapidly cause cardiac decom.pensation with either a fatal termination or surgical intervention.

Surgical intervention should be instituted when the condition is met with. Usually the vessels both above and below can be controlled by detachment of the sterno mastoid just above its origin and the segment of the artery and vein is excised. A time-consuming and difficult operation in a patient whose general condition is far from good.

\section{Late effects of wounds of the lung and pleura.}

These two groups constitute the large percentage of cases producing late effects as a result of thoracic wounds.

Most commonly at the time of the original wound both lung and pleura are involved and maybe both lungs and both pleura, and although at this late stage the affection is chiefly of either lung or pleura, there is nearly always some evidence of the early injury to the alternative structure.

\section{Pulmonary group.}

The outstanding feature of many of these cases, and also with wounds in other parts of the body is the long period of time which has elapsed since the original wound in which the patient has been quite symptomless.

The commonest symptoms are: Hamoptysis, pain and purulent expectoration. 
Hamoptysis varies in amount and frequency within considerable limits, from streaking to 8-Io ozs., and the period of time elapsing between maybe anything from weeks to years. Often hæmoptysis may be the only symptom, the diagnosis of its causation resting solely on the history of a thoracic wound and radiological evidence. When a retained foreign body has been demonstrated radiologically it is of course suspect, but other causes of hæmoptysis should always be kept in mind, as a patient with a retained foreign body is as likely to be heir to other lesions, such as pulmonary tuberculosis and carcinoma as those who have suffered no thoracic injury. In these cases in which a retained foreign body is present, and hæmoptysis is the only symptom, a bronchogram of both lung fields should be done to ascertain the condition of the bronchial tree. At the same time it is possible in a large percentage of cases to demonstrate a communication between the cavity in which the foreign body lies and the bronchial lumen. If there is any doubt as to the foreign body being the cause after bronchography, then bronchoscopy should be performed, and this should also be done in cases where gross bronchial dilatation has been shown.

Pain. Patients with retained foreign bodies will often complain of pain. It is very difficult to assess whether the pain is of a functional character or not. The fact that there is a bullet in the lung is often known and this naturally becomes the focus of his attention and if any question of pensionable disability is involved the condition is aggravated. There can be no doubt however that certain of these cases have definite pain, and the fact that there is no anatomical explanation of it at present is no sufficient argument for denying its existence. Often a satisfactory anatomical explanation can be found when the foreign body lies near the visceral pleura and can reasonably be suspected of irritating the parietal pleura over chest wall, diaphragm, or mediastinum and the distribution of the pain coincides with this hypothesis. In certain cases also it is possible that a mild inflammation may occur around the foreign body, and in certain cases there is evidence that this occurs from the fuzziness in the $\mathrm{X}$-ray picture around the foreign body.

In these cases bronchography should also be performed as well as straight $\mathrm{X}$-rays.

Purulent expectoration: The commonest underlying causes of purulent expectoration are either bronchiectasis or empyema with one or more bronchopleural fistulæ. Occasionally the origin of the sputum may be the cavity around the foreign body with little or no involvement of the bronchial tree.

The sputum may be fœtid or non-fœtid, the factor apparently having no relationship to the extent of pulmonary disease. It is not uncommon in this group to have a history of previous attacks of fever which have been diagnosed as pneumonia or pleurisy, the true underlying cause having been overlooked. These cases often show well marked finger clubbing, the toes often being equally affected.

The patients usually are very toxic with a peculiarly earthen complexion, having lost a great deal of weight. Cough is often troublesome, and sputum is raised more easily when the correct posture is adopted, the posture depending on which portion of the bronchial tree is affected.

Dyspnœa is a variable factor, and this seems to depend on the amount of lung involved together with the amount of pleural involvement and rigidity of the chest wall. Dyspnœea may also be cardiac in origin due to myocardial degeneration resultant of long combined sepsis.

Hæmoptysis may be an accompaniment of the group also, but it is rarely as frequent or massive as in the group in which hæmoptysis is the sole symptom. 


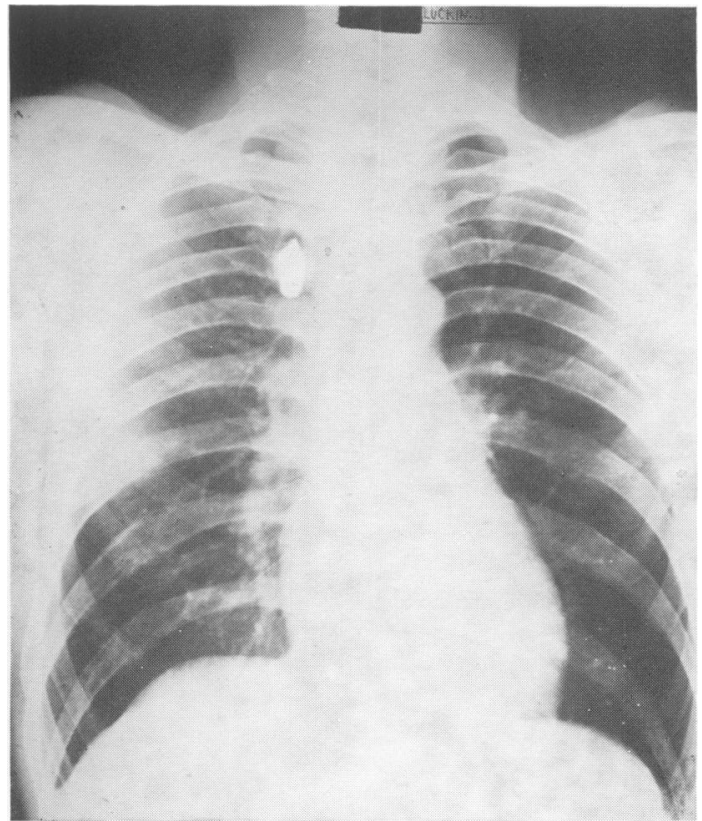

FIG. I.-Antero posterior film shewing gunshot wound (J.W.L.) right upper lobe. Rifle bullet is partially surrounded by shadow due to iron sulphide. This patient had frequent hæmoptyses up to 10 ozs. No purulent sputum. Bronchial tree normal bronchographically.
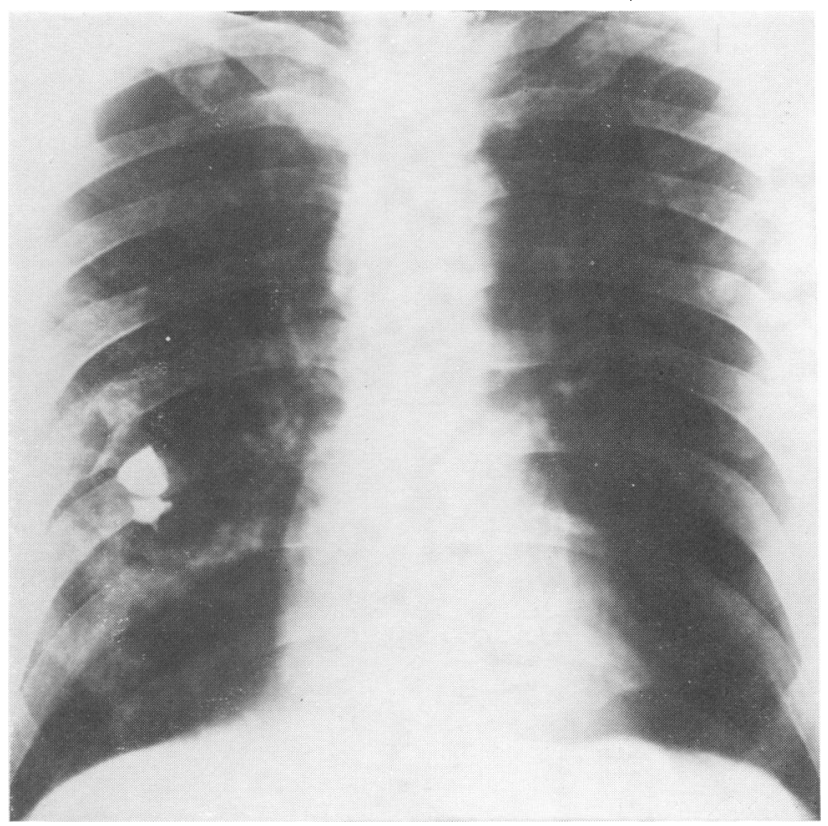

FIG. 3.-Antero posterior film shewing two shell fragments. (W.C.)

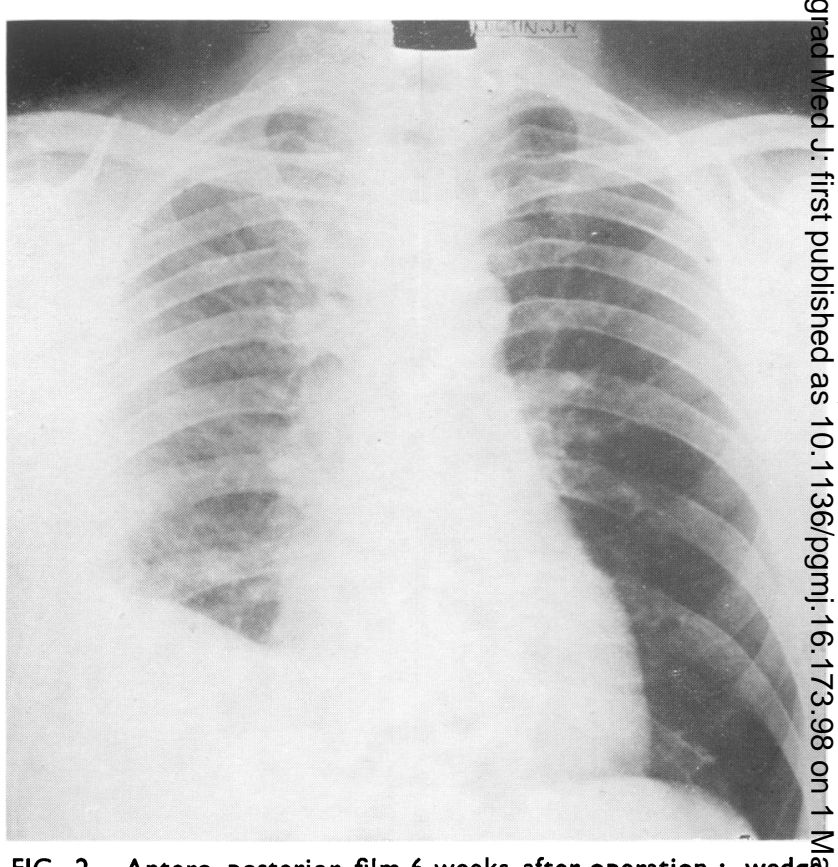

FIG. 2.-Antero posterior film 6 weeks after operation; wedgę (J.W.L.) shaped resection of lung containing bullet.

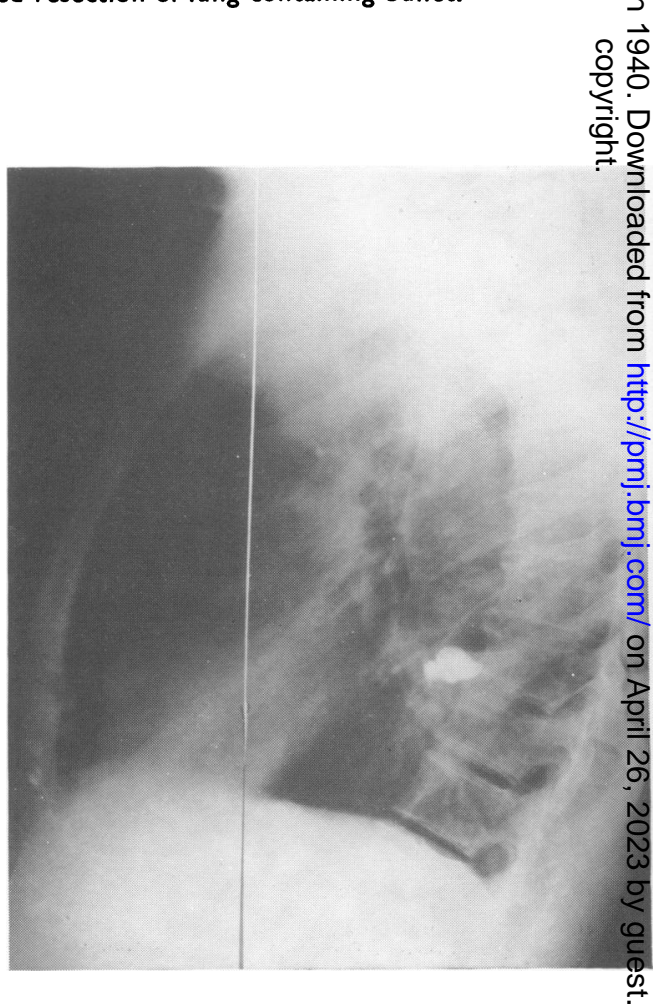

FIG. 4.-Lateral film of same case. Both fragmenty (W.C.) appearing as one, fluid level in cavity also shews in this view. 


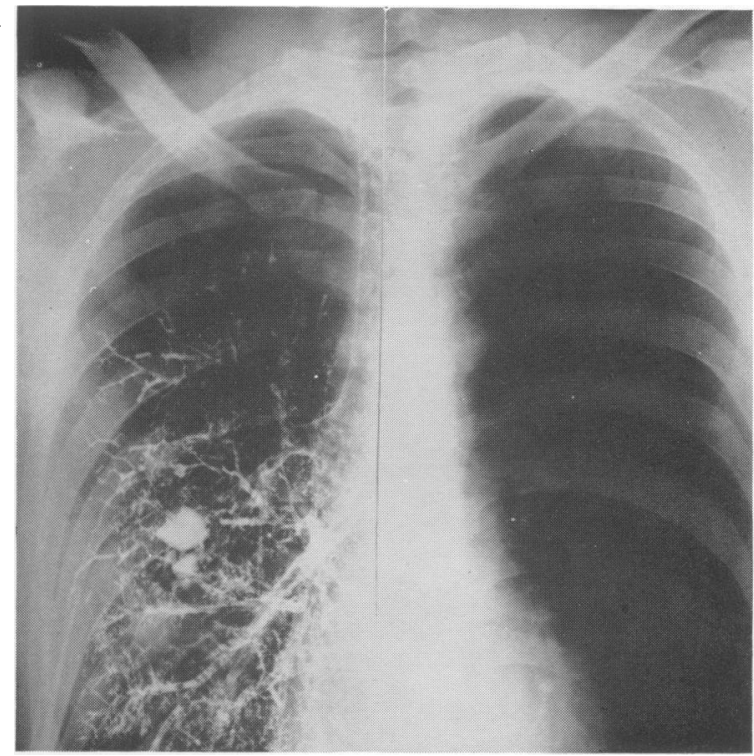

FIG. 5.-Bronchogram shewing absence of bronchiectasis (W.C.) and of cavity filling by iodised oil.

Patient had cough with expectoration of 4-5 ozs. of offensive purulent sputum. No previous trouble for 10 years. Attack commenced with pyrexia and diagnosis of pneumonia until offensive sputum appeared.

Resection of 4-5 ins. of three ribs. Lung adherent, cavity opened in same stage. Cavity about 3 ins. in diameter. Shell fragments, lying loose, were removed.

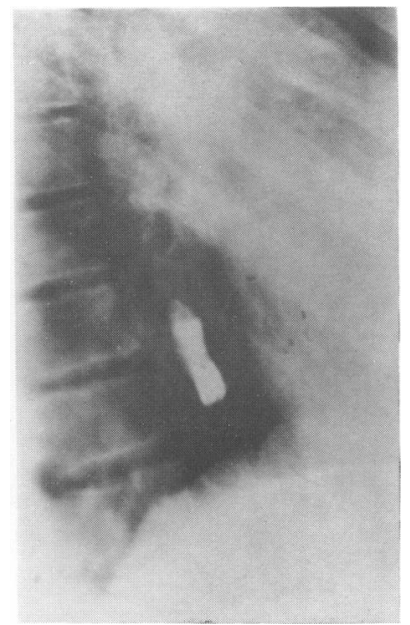

FIG. 7.-Lateral film showing shell fragment behind heart (W.T.P.) shadow in left lower lobe.

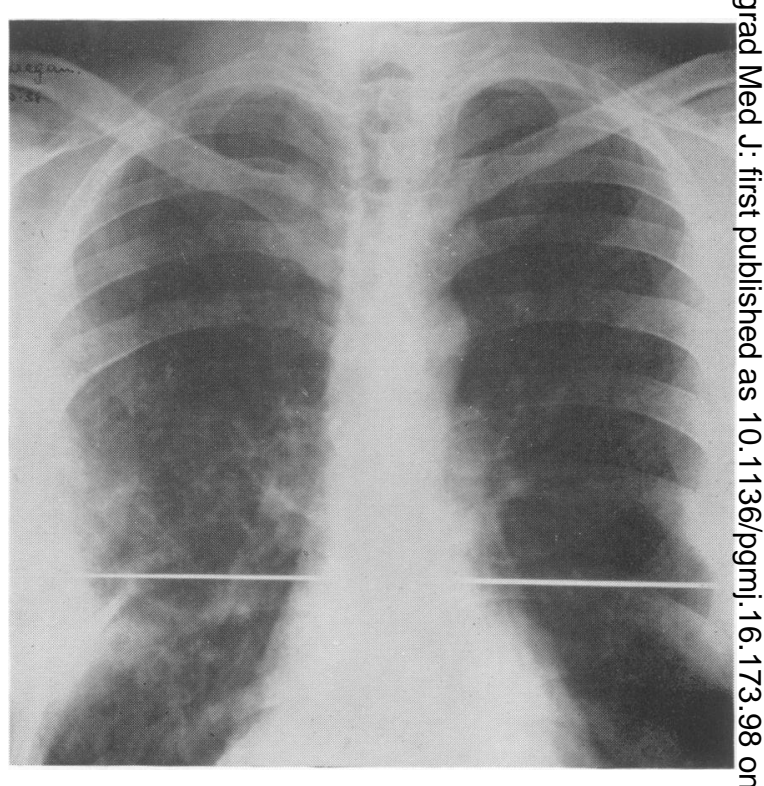

FIG. 6.-Same patient I year later, external sinus healed. (W.C.) Patient still has about 2 drachms of non-offensive $Z$ sputum.

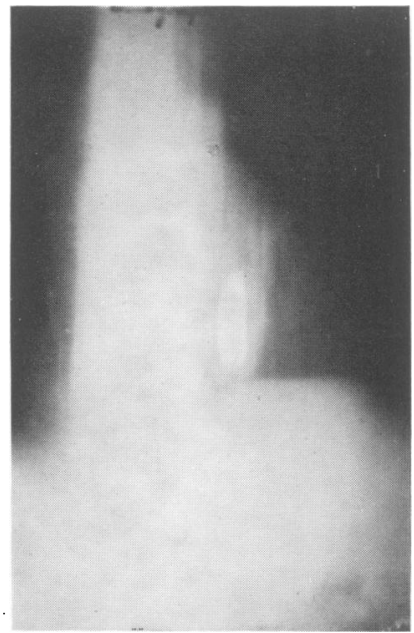

FIG. 8.-Tomographic cut through the foreign body, (W.T.P.) shewing collapsed lung supplied by the paracardiac $\frac{7}{0}$ bronchus.

There was no evidence of bronchiectasis.

The patient complained of cough and purulent expectoration.

At operation lung was found to be adherent. N Lower lobe mobilised from posterior chest wall and pericardium.

Collapsed area of lung demarcated by forceps and $\underline{C}$ divided. Remaining pedicle ligated and divided. Foreign body found lying in smooth walled cavity resembling bronchus. Histological examination suggested bronchial structure.

Patient has now been at work as an engine driver for two years without symptoms, and the $X$-ray is now normal. 
These cases should be investigated clinically and radiologically and if indicated bronchoscopically. Radiological evidence is perhaps the most important.

Sometimes the straight X-ray will afford the most important evidence, a cavity with a fluid level, a collapsed lobe or the presence of foreign body. Bronchography however will practically always be performed. This will show the presence of any dilatation and will also serve to help to differentiate between an intrapulmonary collection of pus and a pyopneumothorax, as often the outer lung limit can be determined by the bronchial filling. It may be mentioned here that it is often difficult to make this differentiation, operation only establishing a correct diagnosis in some cases.

The bronchogram should be a complete one, each lung being filled separately, anteroposterior and lateral views being taken.

\section{Treatment.}

The general overhaul of these patients should be complete before undertaking any treatment of an operative nature. These patients are presenting themselves often a long period after the original wound, and are in the main in the fourth or fifth decade or more, and some of them have been subject to prolonged sepsis, with its deteriorating effect on heart, liver and kidneys. In fact the bulk of these cases provide poor operative risks.

In consequence of this the question of operation should be very carefully weighed against the severity of the symptoms, the rate of the patient's deterioration and the considered prognosis if no intervention is performed.

All possible operations should be done under local anæsthesia and operations should if possible be staged so as to keep well within the patient's powers of toleration. All collections of pus should be drained in the first place, either by open operation or by posture before any more serious operation is undertaken. Blood transfusions are invaluable in dealing with these cases. The chest wall is often very vascular and sometimes by the time the chest wall incision has been completed the unavoidable loss of blood will necessitate the operation being stopped at that point, with an interval transfusion at this time. This help should never be withheld as the hæmopoietic system is as handicapped in these patients as other systems.

\section{Cases with hæmoptysis as the sole outstanding symptom.}

The immediate treatment is conservative; absolute bed rest, morphia and blood transfusions. It is now universally agreed that blood transfusion is the best hæmostatic measure at our command, where the vessel itself is not accessible to control. After this preliminary treatment the investigation of the case is carried out.

Persistent hæmorrhage of a serious enough nature to warrant the consideration of operative intervention has not been met with in the writer's experience; should it be necessary, ligation of the pulmonary artery should be tried. This measure has not been altogether satisfactory when carried out in cases of pulmonary tuberculosis, the difficulty is to decide whether the bleeding is from the pulmonary or the bronchial vessels. Eloesser, in these latter cases has done mass ligation of the pedicle of the lobe, tying the ligature firmly enough to control the vessels, without occluding the bronchus, gangrene of the lobe, contrary to expectation does not occur.

The factors guiding the decision to intervene for the removal of the foreign body are (I) the severity and the frequency of the hæmorrhage; (2) the patient's 
economic status; (3) the general condition and age of the patient. In cases where the hæmorrhages are slight and infrequent there is no call to intervene, but where the hæmorrhages are frequent, the question of removal becomes more urgent, and the second consideration of the patient's economic status comes in for consideration. In this connection if his status is good and he can take requisite care, there being no call for him to continue with a heavy occupation, a conservative course can be adopted, keeping the patient under observation meanwhile. There can be no doubt that the first hæmoptysis does not call for operation even though it be a relatively serious one.

The general condition of the patient carries the same weight as when any operation is under contemplation. The psychological aspect of these patients is important, they have been subject to repeated hæmorrhages, with the concomitant stays in hospital, often with economic factors pressing heavily. It is right to take the patient completely into your confidence and when operation has been decided upon to lay the whole case before him.

In this group infection is clinically absent, and the pleura is relatively free.

Operation when undertaken should aim at removal of the foreign body with the portion of lung in which it is contained. This can only be done in cases where the foreign body is at a sufficient distance from the hilum to allow of a wedge shaped resection of the lung. Removal of the portion of lung with the foreign body is advisable, because organisms often gas formers, can and do live in the cavity walls for years without causing any local disturbance, but are liable to cause an intense reaction when the foreign body has been removed. In cases where the cavity itself cannot be excised completely merely removal of the metal is done. It is probably unwise to use diathermy for the purpose, as by doing so dead tissue is left which forms an ideal pabulum for these organisms. One other reason for excision of the surrounding lung is that the bleeding points will be more certainly controlled. A pneumothorax is done preoperatively, and this will also show the extent of pleural adhesions. The chest is opened by an intercostal incision. The foreign body is located and if it is located in such a position as to allow resection, an intestinal clamp is placed so as to control the lobe, proximal to the area of resection. This is necessary to prevent if possible the occurrence of air embolism. With this satisfactorily in position the pulmonary vessels are controlled and generally the bronchi as well. The wedge shaped area to be removed is now demarcated with crushing forceps, long bladed Spencer Wells serve very well, the area is packed off and the wedge is resected and a running hæmostatic suture is placed over the forceps on each side, great care must be taken to effectually seal the apex of the wedge. If the wedge is large mattress sutures parallel and proximal to the forceps are placed, one suture overlapping the other in order to ensure that the whole area is included, and then opposing surfaces of the wedge are approximated in the same manner as a wedge resection of the liver is dealt with. The intestinal clamp is now removed and the resected area carefully inspected for air or blood leak, in the large resection the clamp is of course removed before the edges of the wedge is approximated. The chest is now closed in layers, an intercostal tube having been inserted before the chest is closed. The tube serves the purpose of a safety valve should a bronchus open and so prevents a tension pneumothorax and also drains the serous exudate which invariably occurs. It can be removed in three to four days unless there is an indication for leaving it, such as pleural sepsis. Pleural involvement however does not usually occur in these cases, and if it does generally takes nine to ten days to develop. When it does so, the intercostal tube should be replaced, if it had previously been removed. 
In cases where there is a complete pleural symphysis the incision should be planned to lie over the foreign body, which has been localised previously, generally by means of the anterior, posterior, and lateral films, sometimes however tomographs will be needed in cases where the foreign body is in the upper part of the upper lobe and the shoulder girdle prevents a good lateral.

In these cases it is better to resect three to four inches of the rib overlying the piece of metal. After the pleura is incised it is possible sometimes to get enough mobilisation of the lung to carry out the previous technique in a modified way, if not the lung is incised over the foreign body which is removed. The localisation of the metal can be extremely difficult when the limited approach has to be used, as if the lung cannot be freely handled it is difficult to detect it; it is often difficult enough with the smaller pieces of metal even when the lung can be freely palpated. When the foreign body has been removed, it is safe to drain preferably by lightly packing the cavity with paraffin gauze in the first instance; a tube may cause pressure on vessels and lead to delayed hæmorrhage. Later, tube drainage is instituted and the tube is removed when discharge has ceased and the fistula is closed. Sepsis always accompanies this method of removal.

\section{Cases complaining of pain only.}

There is rarely any indication to operate in these cases, however there are occasions where pain is so persistent, and the patient so importunate, as to demand an operation. A fair general rule can be made in cases where the foreign body is centrally placed, as opposed to those situated at the extreme periphery of the lung, that when a bronchogram has been done, the opaque oil goes into the cavity surrounding the foreign body, showing that the cavity is in communication with the bronchial tree. If a communication with the bronchial tree cannot be established, then it is not justifiable to undertake surgical removal, as a foreign body may be perfectly encapsulated with fibroid tissue and in these circumstances can be of no menace to the patient, it is the communication with the bronchial tree, by keeping the way open to infection which causes the hæmoptysis, pneumonitis and purulent sputum.

\section{Cases with purulent sputum.}

The cause of the purulent sputum may be bronchiectasis or a pulmonary abscess around the foreign body; in the latter case there is always a varying degree of bronchiectasis. The treatment in this group will depend on the type of lesion and the extent of the disease. Obviously the symptoms and prognosis must be sufficient to warrant the risk of the operative intervention. The patient with a few symptoms and little pathological change as shown radiologically and bronchographically will not warrant an operation, as at this late stage the rate of progress of his disease will probably not be much faster than his deterioration as a result of increasing age.

In cases where the symptoms are severe with their attendant physical and economic disability, there can be no question that operation is the treatment of choice, providing the patient is in a fit state to support it.

The majority of cases however lie between these two extremes. The problem of some cases will be relatively easily solved in favour of operation, but when any doubt exists a conservative course should be adopted and postural drainage employed. The patient can be reassessed in three to six months, when an estimate can be formed as to progress in one or other direction. The above remarks apply chiefly to those cases with an accompanying bronchiectasis. 


\section{Foreign body with an associated pulmonary abscess.}

These cases most often present themselves in an acute or a sub-acute stage. The evidence of the existence of the abscess is nearly always obvious by X-rays, doubtless due to the fact that the small cavity originally surrounding the foreign body always was in communication with the bronchial tree, allowing of easy rupture of the abscess into a bronchus, with admission of air into the cavity.

These cases require operation. Preoperative postural drainage, in the correct position to drain the cavity, should be started at once if the patient is fit enough to tolerate it, and it is likewise well to do a bronchogram if circumstances permit, to ascertain if there is a gross associated bronchiectasis.

The cavity is localised with anteroposterior and lateral films and under local anæsthesia lengths of two or three ribs are resected over it. If localisation is accurate the lung is always found stuck to the chest wall; if however there is any doubt of this, an iodine pack is placed on the pleura, which has been cleared of the intercostal bundles and the wound is closed in layers. Ten days later the wound is reopened when the pleural surfaces are adherent, the cavity is explored with a needle which is left in situ when pus is aspirated and the cavity is then opened along the needle with knife or diathermy; the latter is preferable if the lung overlaying the cavity is vascular. The cavity is now explored under vision and the shell fragment is removed. As much of the roof of the cavity as possible is removed, all adjacent loculi if present are opened into the main cavity which is then packed lightly with paraffin gauze. The superficial wound and entrance to the cavity is similarly treated with packing and this packing remains in situ for five to seven days and is repeated for as long as is practicable, this may be four or five weeks when a tube is inserted, and this is maintained until the cavity closes and the fistula heals. The prospect of the cavity closing is not as bright as with the ordinary lung abscess, as their walls are generally fibrotic and hard and further treatment by lobectomy may have to be considered, or else the tube must be persisted with.

In certain cases of abscess, as has been indicated, there is present as well a gross bronchiectasis. The abscess drainage is first instituted and the case then is considered from the point of view of the bronchiectasis present.

\section{Bronchiectasis associated with pulmonary foreign bodies.}

In the group of cases under consideration the bronchiectasis does not develop by virtue of bronchial occlusion by the foreign body as occurs with inhaled foreign bodies. The underlying factor producing the bronchiectasis in these cases is the sepsis engendered around the foreign body. In other words the bronchiectasis is secondary to the abscess around the foreign body, but in these cases the abscess cavity is very small and as a clinical entity is insignificant. In some cases the foreign body may not be present, the bronchiectasis being a direct sequela of sepsis occurring at the time of the original wound, or from a long standing chronic empyema, with or without a bronchial fistula, in which the lung itself has partaken, at some time during its course, in the infective process. Occasionally there may be no other points in the history other than that of the wound with hæmoptysis at the time, and a bronchial block with low grade infection may have occurred at the time of the original wound as a result of aspirated clots. The bronchiectasis may be lobar, or more than one lobe may be affected. The dilatations are nearly always fusiform in type. The treatment of this group of cases follows the lines 
of the treatment of any case of bronchiectasis by lobectomy or pneumonectomy, always keeping in mind that the age group is higher.

Complications of operations in this type of case.

(I) Air Embolism. This complication has already been indicated and measures taken for its avoidance.

Air embolism classically is a very dramatic event whether it results from the admission of large quantities of air through one of the large veins such as the internal jugular or axillary, or as a result of air introduced into small veins under pressure as during aero-urethroscopy. In this type of case however it may be anything but dramatic in that the patient may survive for $24-36$ hours after operation before a fatal termination. This possibility is the result of a slow but constant leak, or maybe that the leak results from expansion of the lung after closure of the chest with rupture of the pulmonary suture line. In any case the fact that it is slow and the outcome occurs long after the operation is finished and the site of the leakage is consequently inaccessible, makes it imperative that the primary suture should be above suspicion at the time of its introduction, no care being spared to ensure sufficient control of the bronchial and vascular elements.

(2) The lighting up of dormant gas infection. There is definite risk of stirring up the activity of gas infections after many years when operating on patients with retained pieces of shell or rifle bullets wherever they may be lodged in the body and the same risk applies to these cases. When a foreign body has been removed the lining of the wall is deeply stained with iron sulphide in cases where there is no obvious inflammatory process, but in the course of a day or so there is a fairly profuse foul discharge from the cavity and in one personal case the patient developed a septicæmia and died. In this case the lung had been incised with diathermy and it was thought that more extensive destruction of tissue than the mere incision, was produced by induction through the foreign body itself, with a resulting increase in the area infected beyond the boundaries of natural defence which had already been built up in the walls of the cavity.

The other complications are those to be met with in the course of any chest operation, such as empyema, spread of infection to other parts of the lung fields, pericarditis, etc., and need no special mention here.

Affections of the pleura.

The late effects on the pleura are chronic empyemata, and chronically thickened pleura, the former being the commoner. The cases with chronically thickened pleura can occur without any demonstrable associated empyema in cases where the sole antecedent has been a hæmothorax which has been treated by aspiration. Brock in a personal communication states that he has seen calcification in the pleura without any evidence of empyema; this must be exceptional however, as the presence of deposition of calcium in the pleura generally means that an empyema is present even though drainage has not been established. This marked thickening of the pleura constitutes a serious disability to the patient; acting as it does like a vice, holding the hemithorax immobile, and in consequence limiting seriously his vital capacity. There is very little in the way of treatment which can be done for these cases when the condition is established, the appropriate moment for treatment is earlier at the time of the original injury.

Chronic empyema is the commonest pleural involvement to be met with and is the commonest disability to be met with as a result of thoracic injuries. 
The large majority of these cases have already had drainage operations before, some of them are still discharging externally, have been doing so constantly for twenty years, others discharge intermittently, others may be coughing the empyema up through bronchopleural fistula, while others may have an encapsuled empyema without any external outlet.

The cases with external discharge are usually easy of recognition, but occasionally the case with intermittent discharge is overlooked. Often the period of discharge is of short duration, the sinus leading to the empyema very tortuous and in consequence the diagnosis of necrosis of rib is made and the underlying empyema is completely overlooked. It can be safely said that an empyema wound which repeatedly breaks down and discharges is almost inevitably covering a chronic empyema and in consequence the case should be thoroughly investigated radiographically and by exploration of the sinus, if necessary surgically.

When an external sinus is present it should be enlarged so as to allow of a reasonable size tube to be inserted. Laminaria tents are best used for dilatation of the sinus, the largest size possible being inserted first and left until a decent size tube can be introduced, slow dilatation by this method is much better than rapid dilatation with bougies, the risk of dislodgment of thrombi is considerably lessened and it is much less painful for the patient. When the track is dilated, iodised oil is introduced into the cavity and X-rays taken in anteroposterior and lateral planes.

Occasionally it is impossible to dilate the sinus and in these cases surgical exploration will be necessary to locate the cavity or follow up and lay open the track.

In cases with a bronchopleural fistula the straight X-rays will often establish the diagnosis but as already stated it is difficult to determine whether the fluid level shewn is intrapulmonary or intrapleural except by operation. Valuable information can be gained by the introduction of some iodised oil into the pleural cavity with an exploring needle, this will give the lower limit of the pocket and a definite indication for the site for drainage so that it shall be dependent.

In the closed cases the condition is usually discovered radiographically, the patient generally complaining of pain, dyspnœa, general debility and of repeated attacks of pyrexia which have been diagnosed variously as either pleurisy, pneumonia, or some other condition which is totally unrelated to the chest: the majority of these patients shew finger clubbing, some to an extreme degree and this when observed in a patient, especially one who has suffered from a chest wound should immediately lead to the suspicion of an intrathoracic focus of suppuration. All of these cases may show evidence of calcification of the pleura radiologically and this can often also be felt by a probe which sometimes confirms the unwary in the diagnosis of necrosis of a rib.

Some consideration must be given to the origin of this group of cases. The majority of them developed empyemata at the time of the wound, and may be considered as chronic empyema superimposed on the acute variety. Others however are superimposed on a hæmothorax which has been inefficiently dealt with. It is well known that certain cases of hæmothorax will react to conservative treatment by aspiration in a most gratifying manner, while others behave in anything but a satisfactory way and the crux of the situation appears to be whether or not the blood remains fluid. If a large coagulum forms then it is more than probable that some disability ensues, the result being either organisation of the coagulum, 
which probably explains the cases described earlier of thickened pleura, or infection of the coagulum with a consequent empyema which manifests itself in any of the above ways.

The acute cases which have become chronic have in the main occurred as a result of inappropriate treatment in some particular, either that drainage has been dispensed with before the cavity has been obliterated, or the drainage site has been unsuitably placed. In this group there is rarely an intrapulmonary cause which prevents re-expansion of the lung, although this should always be kept in mind.

In the treatment of these cases, the prime essential is adequate and well placed drainage, and this by itself is often sufficient to ensure closure of the cavity. The rate of closure can be increased by establishing negative pressure drainage by means of an aspirating pump up to a pressure of $5-20 \mathrm{cms}$. of mercury. Control by X-rays with iodised oil in the cavity is essential and the tube is not to be discarded until X-ray demonstration shows the cavity to be properly closed. Occasionally cavities will not close even with negative pressure drainage. In cases where the cavity is relatively small the ribs overlying the cavity are removed generously, and the roof of the cavity is excised, the remaining wound being packed and allowed to heal by granulation. Cases where there is no cavity but merely a series of sinuses are similarly treated, the greatest care being taken to follow the sinuses to their termination; this often proves to be a very tedious proceeding.

Where there is a large cavity, especially so in the presence of bronchial fistulæ, which after efficient drainage does not decrease in size, the operation devised by Roberts is very efficient. The whole cavity area is thoroughly and generously decostalised, this is done in stages through the same wound and the parietal pleura at the last stage is mobilised as a hinged flap with the hinge posteriorly, preserving the intercostal vessels as blood supply to the flap, which with the parietal pleura is cleared of granulations by dry swabbing. The flap is then packed down on the lung surface and held thereby by a gauze pack over which the musculo-cutaneous flap is sutured. The wound is reopened in a week or ten days and the flap examined to see if it is adherent, if not a fresh pack is put in place and the wound is resutured and reopened in a further week which usually suffices for the flap fixation. The wound is closed and an extra cutaneous pad is fixed over the area.

These operations should be planned in stages so that they can be stopped at any time. The chest wall in these cases is very vascular and a great deal of blood may be lost. The patients also are often feeble and their resistance much reduced, and there should in consequence be no attempt to complete the operation in one stage, however great the temptation, if there is the slightest suspicion that the patient will be badly knocked out by doing so. The best indication is a rise in pulse rate and a fall in blood pressure, and a change of twenty in either should be taken as a sign that the patient's toleration for that session is being reached. Close co-operation between the operator and the anæsthetist is essential and the latter should be experienced in managing the type of case.

\section{Conclusions.}

The study of these late results lead one to draw certain conclusions as to the early treatment at the time of injury which may be briefly stated.

Firstly, that many cases who survive the original wound and for many years live free from any obvious disability in this connection, do at a later date develop 
conditions which carry with them a high mortality rate and present difficult and serious problems for treatment at this late date.

It seems from the consideration of this group, that with proper facilities, surgical, anæsthetic, and nursing, that penetrating wounds of the lungs and pleuræ when the foreign body is retained and when there is a hæmothorax present, should be subject to deliberate thoracotomy with, if possible, resection of the quadrant of lacerated lung including the foreign body, efficient hæmostasis of the chest wall wound and primary closure of the chest with temporary drainage.

The immediate treatment of hæmothorax should depend on the result of primary aspiration. If no coagulum is demonstrable after aspiration, then that treatment should be persisted in. If there is a coagulum then the chest should be explored early before infection has had time to occur, the coagulum cleared out and the chest wall injury dealt with. The presence of a large coagulum would tend to indicate a fair degree of laceration of the inner aspect of the chest wall or lung surface as without a reasonable admixture of tissue fluids, the blood in the pleura would remain fluid.

Lastly, when empyemata occur at the time of the original injury, no trouble should be spared in ensuring that the lung is fully expanded and the cavity completely closed before drainage is discarded.

I am grateful to Dr. H. Lightstone, D.S.O., M.C., Acting Director-General of Medical Services of the Ministry of Pensions, for permission to use the X-ray reports of cases in Queen Mary's Hospital, Roehampton. 